
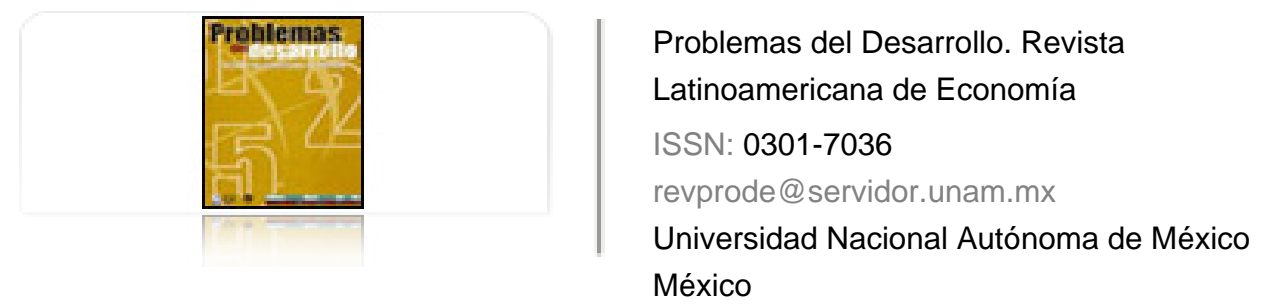

García Garnica, Alejandro; Taboada Ibarra, Eunice L. La coordinación de recursos en la industria de asientos automotrices: el caso de Lear Tláhuac Problemas del Desarrollo. Revista Latinoamericana de Economía, vol. 36, núm. 143, octubrediciembre, 2005, pp. 171-187

Universidad Nacional Autónoma de México

Distrito Federal, México

Disponible en: http://www.redalyc.org/articulo.oa?id=11820081008

- Cómo citar el artículo

- Número completo

- Más información del artículo

Página de la revista en redalyc.org

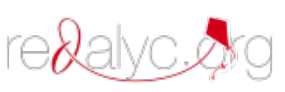

Sistema de Información Científica

Red de Revistas Científicas de América Latina, el Caribe, España y Portugal Proyecto académico sin fines de lucro, desarrollado bajo la iniciativa de acceso abierto 


\section{LA COORDINACIÓN DE RECURSOS EN LA INDUSTRIA DE ASIENTOS AUTOMOTRICES: EL CASO dE LEAR TLÁHUAC*}

\section{Alejandro García Garnica** Eunice L. Taboada Ibarra***}

Fecha de recepción: 18 de febrero de 2005. Fecha de aceptación: 10 de octubre de 2005.

\section{Resumen}

Con la finalidad de acceder a nuevos conocimientos e información, aumentar sus capacidades tecnológicas, diversificar su producción o introducirse en nuevos mercados, las empresas de la industria automotriz han variado la forma de coordinar sus recursos: utilizan alianzas estratégicas, la integración vertical o la modularización organizacional. El objetivo de este trabajo es describir y analizar las estrategias de coordinación que han seguido algunas empresas de la industria de asientos automotrices en el nivel mundial y, en particular, la planta Tláhuac, de Lear Corporation.

Palabras clave: México, automotriz, modularización, coordinación, asientos automotrices.

\section{Abstract}

Aimed at gaining access to new know-how and information to increase their technological capacities, diversify their production or gain entry to new markets, auto industry companies have changed the way they coordinate their resources: they are using strategic alliances, vertical integration or organizational modularization. This study aims to describe and analyze the coordination strategies followed by some companies in the automobile seating industry worldwide and, in particular, Lear Corporation's Tláhuac plant.

Key words: Mexico, automobile, modularization, coordination, automobile seating.

* Este trabajo se deriva de la tesis doctoral de Alejandro García en Estudios Organizacionales, "Coordinación inter e intra empresa en la industria automotriz de autopartes: los casos de algunas plantas de Lear Corporation”, Universidad Autónoma Metropolitana, Unidad Iztapalapa, octubre, México, 2004. Agradecemos los comentarios y sugerencias que amablemente nos hicieron tres dictaminadores anónimos asignados por la revista; la responsabilidad de las ideas expuestas en este trabajo es sólo nuestra.

** Profesor investigador de la Universidad Autónoma del Estado de Morelos, campus oriente. Correo electrónico: agg67@hotmail.com

*** Profesora-investigadora del Departamento de Economía de la Universidad Autónoma Metropolitana Azcapotzalco. Correo electrónico: elti@correo.azc.uam.mx 


\section{ALEJANDRO GARCÍA GARNICA Y EUNICE L. TABOADA IBARRA}

\section{Résumé}

Afin d'accéder à des connaissances et des informations nouvelles, d'augmenter ses capacités technologiques, de diversifier sa production ou de s'introduire dans de nouveaux marchés, les entreprises de l'industrie automotrice ont modifié la manière de coordonner leurs ressources: elles utilisent des alliances stratégiques, l'intégration verticale ou la modularisation organisationnelle. L'objectif de ce travail est de décrire et d'analyser les stratégies de coordination de quelques entreprises de l'industrie de sièges automotrices au niveau mondial, et plus particulièrement dans le siège de Tláhuac de Lear Corporation.

Mots-cléfs: Mexique, automobile, modularisation, coordination, sièges automotrices.

\section{Resumo}

Com a finalidade de obter novos conhecimentos e informação, acrescentar suas capacidades tecnológicas, diversificar sua produção e se introdizir em novos mercados, as empresas da indústria automotriz têm variado a forma de coordenar seus recursos: utilizam alianças estratégicas, a integração vertical ou a modularização organizacional. O objetivo deste trabalho é descrever e analizar as estratégias de coordenação que seguiram algumas empresas da indústria de assentos automotrizes no nivel mundial e particularmente a planta Tláhuac de Lear Corporation.

Palavras chave: México, automotriz, modularização, assentos automotrizes. 


\section{Introducción}

$\mathrm{E}$ 1 paradigma de organización y producción flexible que se ha extendido y adaptado en gran variedad de empresas automotrices asiáticas, americanas y europeas es una respuesta al conjunto de cambios sociales y tecnológicos que afectan a ese sector (Womack, et al., 1990).

Las transformaciones y requerimientos a las cuales ha dado lugar este paradigma han incrementado la competencia al racionalizar los procesos productivos (reducir los costos de ensamble y de coordinación), aumentar la velocidad de respuesta ante las variaciones en la demanda de los consumidores (Helper, et al., 1999, y Freyssenet y Lung, 1996) y acortar el ciclo de vida de los productos y procesos automotrices (Fine, 2000). Asimismo, a la flexibilidad del proceso se ha sumado el crecimiento de fusiones y adquisiciones y el aumento de las relaciones de colaboración entre las ensambladoras, y entre éstas y sus proveedores.

Uno de los factores que ha contribuido al impulso de la diversidad de relaciones interempresariales es la globalización de la economía; ésta ha significado la integración y la coordinación funcional de distintas actividades productivas y de servicios que componen la cadena de valor de un producto y se encuentran dispersas en distintos países y regiones (Gereffi, 2002).

La integración de la producción mundial ha propiciado que las empresas, especialmente las multinacionales, establezcan distintas formas de vinculación con sus proveedores ante la necesidad de: a) diversificar sus fuentes de abastecimiento de recursos humanos, materiales y financieros (Cohen, 1981 y Shimokawa, 1999); b) incrementar sus economías de escala; $c$ ) acceder a nuevas tecnologías, y $d$ ) satisfacer a tiempo las necesidades de sus clientes.

Con el fin de mostrar distintas formas de coordinación de recursos que el entorno competitivo exige a las empresas, en este trabajo se ejemplifica el caso de la industria de asientos automotrices. Para tal efecto, se describen algunas de las estrategias que han seguido los principales actores de este mercado y, mediante el estudio de la planta de Lear Corporation en Tláhuac, se ilustra y analiza la manera en la cual ésta se vincula y coordina con otras plantas filiales y con sus clientes en México. Particularmente se destaca cómo el corporativo logra que las plantas cooperen/compitan, pero a la vez mantengan una dependencia/independencia relativa.

Las preguntas básicas por responder son: ¿qué están haciendo las empresas líderes de la industria de los asientos, particularmente Lear Corporation, para diversificar su producción

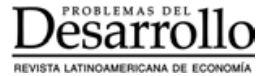


y acceder a recursos y conocimientos que no poseen internamente?, ¿cómo se relaciona Lear Tláhuac con las plantas que integran su corporativo y cómo se vincula con sus clientes?

El trabajo está estructurado en dos partes. En la primera, brevemente se exponen las formas de coordinación de algunas empresas líderes de la producción de asientos automotrices. En la segunda, se presenta un estudio de caso: Lear Tláhuac, planta productora de asientos y puertas y de algunos componentes del sistema de interiores. Se describe la importancia de los procesos de cooperación y coordinación de recursos humanos interplanta para que ésta se diversifique, así como el tipo de relaciones de colaboración entre Lear Tláhuac y las ensambladoras.

\section{Formas de coordinación en la industria de asientos automotrices y en Lear Corporation}

En la actualidad no hay una tendencia general respecto de las formas de coordinación en la industria automotriz. Mientras algunas organizaciones productivas ${ }^{1}$ han aumentado sus capacidades tecnológicas mediante fusiones o adquisiciones, otras empresas han optado por crecer internamente (Álvarez, 2002). También es común el establecimiento de diferentes formas de vinculación o asociación interempresariales, tales como inversiones conjuntas, acuerdos de investigación y desarrollo, contratos de transferencia tecnológica, licencias, entre otros.

Al respecto, Womack, Jones y Roos (1990) señalan que en la industria automotriz existe la tendencia a adoptar tres formas:

a) La centralización extrema de la toma de decisiones en las sedes matrices, las cuales se sitúan regularmente en el país de origen. Éste fue el enfoque que Ford impulsó, desde 1908 hasta los años setenta, y es una estrategia seguida por muchas empresas japonesas instaladas fuera de su territorio.

b) La descentralización extrema hacia distintas regiones de las filiales que integran el corporativo. Cada planta desarrolla sus propios productos, sistemas de fabricación y planes de carrera profesionales y mantiene vínculos débiles con las otras empresas localizadas en otras regiones. Ésta fue la postura de Ford en Europa en los años setenta y describe la práctica que General Motors aún tiene en el mismo continente.

c) Las alianzas estratégicas o diversas formas de cooperación interempresa.

Las organizaciones productivas son entidades socioeconómicas que tienden a especializarse y, en esa medida, requieren establecer relaciones permanentes de colaboración comercial y productiva con otras empresas o instituciones. La coordinación de esos recursos puede darse por medio de precios, contratos, autoridad, rutinas, estándares y mediante la confianza (García, 2004).

\section{DeSarrollo}


La forma específica de coordinación de recursos que eligen dos o más empresas —para enfrentar la competencia, altos costos asociados a la investigación y desarrollo tecnológico, así como la incertidumbre económica del mercado automotor- están ligados a: 1) la acumulación y fortalecimiento de capacidades y procesos de aprendizaje de las partes negociantes; 2) la estrategia que ha seguido cada empresa; 3) las necesidades y el potencial de sus recursos financieros, humanos y materiales; 4) la complejidad de los procesos o productos que desean desarrollar; 5) las restricciones gubernamentales, por citar solamente algunos elementos (García, 2004). Es decir, en cada rama industrial, las empresas buscan la manera más eficiente de adaptarse al medio ambiente y de acceder a los recursos que requieren de acuerdo con su propia historia y capacidades.

Algunas modalidades de colaboración o de alianzas estratégicas entre empresas son: las joint ventures (empresas conjuntas), subcontrataciones, licencias, redes y fusiones (Penrose, 1962). En la industria automotriz, esas redes de cooperación han atravesado fronteras regionales y nacionales; por ejemplo, General Motors tiene acciones de Suzuki Motors Co.; Ford Motors Company colabora con Mazda Motors Corp. en diversos proyectos automotrices, y Daimler-Chrysler tiene acciones con Mitsubishi Motors Company, e incluso le compra motores para coches pequeños (Suzuki, 1999).

En la industria de autopartes automotrices sucede algo similar. Beechman (2001) y Aroq Limited (2002) proporcionan algunos ejemplos respecto de las distintas opciones de coordinación y cooperación observadas en la industria de los asientos:

1) Johnson Controls e Ikeda Bussan realizaron en 1986 una joint venture con el fin de fabricar asientos para la planta de Nissan en Estados Unidos. Johnson Controls también realizó otra en ese país, con una planta de asientos de Tachi-S para suministrarlosa Honda Manufacturing y a Saturn.

2) En 2001, Magna fusionó tres de sus principales grupos de negocios (Magna Interiors, Magna Seating y Atoma Closure Systems) con una sola identidad llamada Inter Automotive. Posteriormente, junto con Namba usA Corporation (subsidiaria de una compañía japonesa) crearon Bloomington-Normal Seating Company para manufacturar el módulo de asientos para Mitsubishi/Daimler-Chrysler en Estados Unidos.

3) En 2000, Faurencia adquirió Sommer Allibert's Automotive; esta última era líder europeo en la producción de módulos de puertas, sistemas acústicos y tableros frontales. En 2001, Faurencia realizó un acuerdo de colaboración con NHK Spring Co. para fabricar de manera conjunta asientos para Nissan Motor y, ese mismo año, Faurencia también acordó un contrato de cooperación con Namba Press Works con el fin de fabricar asientos para Mitsubishi y Chrysler.

\section{DeSarrollo}


Por su parte, Lear Corporation, uno de los principales líderes en la producción mundial de asientos, ha optado por comprar ${ }^{2}$ gran número de empresas relacionadas con el negocio de los sistemas de interiores. Se calcula que desde 1994 hasta 2002 este corporativo llevó a cabo un total de 17 adquisiciones estratégicas en el mundo con el fin de desarrollar y acumular capacidades tecnológicas que le permitieran ofrecer a sus clientes un portafolio completo de productos y servicios (Lear Corporation, 2000).

Mediante dichas adquisiciones, Lear Corporation pudo comprar la línea Automotive System Seating de Delphi, una de sus principales competidoras en el nivel mundial. Lo hizo también con Borelis Industrie AB — productora de paneles de instrumentos—y Donelly Corporation Overhead System - fabricante de techos para interiores-. De igual modo, se adueñó de Dunlop Cox y Seat Sub-Systems Unit, las cuales fabricaban diversos mecanismos de ajuste y peso para asientos (Beecham, op. cit.).

Sin embargo, la apropiación más importante de Lear Corporation (1999) fue la de UT Automotive Inc. (por la cual pagó más de dos billones de dólares) y ha sido muy significativa para su desarrollo tecnológico, porque el sistema eléctrico-electrónico es el núcleo neuronal de todos los módulos (puertas, asientos, techos y tableros) ${ }^{3}$ que integran los interiores del automóvil (Lara, García y Rivera, op. cit.).

Las adquisiciones de Lear Corporation le permitieron expandir su negocio, incrementar el número de plantas que potencialmente tienen la capacidad para controlar los costos, satisfacer las necesidades de sus distintos clientes y ampliar rápidamente las plataformas productivas que añaden valor a los distintos bienes que esta empresa fabrica (Lear Corporation, 1999).

En México, Lear Corporation ha realizado importantes compras, las cuales le han permitido diversificar su producción y ampliar sus capacidades productivas. Sin embargo, como se detalla en los siguientes apartados, la incorporación de nuevos recursos humanos y materiales, así como la asimilación de procesos y tecnologías, exigen gran esfuerzo de coordinación y cooperación de recursos humanos en el nivel interplanta.

2 En el caso de la industria de asientos para automóviles, Lear Corporation, Johnson Controls y Magna International son los principales corporativos estadounidenses productores de asientos y sistemas de interiores. Las tres empresas concentran $91 \%$ de los 9.2 billones de dólares que anualmente se venden en ese mercado: Lear Corporation concentra $41 \%$, Johnson Controls, $42 \%$, y Magna International, 8\% (Aroq Limited, 2002). Sin embargo, se ha calculado que en ese país hay alrededor de 23 empresas dedicadas a la fabricación de asientos automotrices completos (Beecham, 2001). Para mayor información acerca de empresas que integran esa rama industrial, véase Lara, García y Rivera (2002).

3 Baldwin y Clark (2000:63) señalan que un módulo: "is a unit whose structural elements are powerfully connected among themselves and relatively weakly connected to elements in other units. Clearly there are degrees of connection, thus there are gradations of modularity".

\section{DeSarrollo}




\section{Relaciones de colaboración de Lear Tláhuac con sus plantas filiales y las ensambladoras automotrices}

Para ubicar a Lear Tláhuac como parte de Lear Corporation y comprender mejor sus estrategias de coordinación y colaboración de recursos, primero se presenta la trayectoria de esa planta y, después, las estrategias de coordinación de recursos con sus clientes internos (las otras plantas) y externos (las ensambladoras automotrices).

\section{Algunas características y evolución del corporativo Lear en México}

Lear Corporation es una empresa estadounidense fundada en 1917 para ensamblar y soldar autopartes. En la actualidad, fabrica distintos componentes que integran el sistema de interiores de automóvil y entre sus principales clientes se encuentran General Motors, Ford, Volkswagen, Daimler-Chrysler y Nissan, por citar algunas importantes ensambladoras. Cuenta con más de 100 mil empleados y 300 plantas distribuidas en todo el mundo.

En México, el corporativo Lear se divide en tres. La primera es la División de Interiores (Manufacturing Operations Division), encargada de manufacturar toldos, viseras y paneles de puertas. La segunda es la División de Asientos (Seat Division), dedicada al cosido, tapizado y herrería de asientos automotrices. La tercera se denomina División de Sistemas Eléctricos (Lear Electrical Systems de México), responsable de ensamblar y maquilar los arneses eléctrico/electrónicos para diferentes módulos automotrices.

En 1995 prácticamente no existía la División de Sistemas Eléctricos en Lear México; ninguna de sus unidades fabricaba arneses. Las plantas que le pertenecían eran solamente diez, ocupaban a casi siete mil personas, de las cuales $67 \%$ se concentraban en una sola entidad (Ciudad Juárez, Chihuahua). De dichas plantas, tres eran maquiladoras (Favesa III, IV y v), se dedicaban a asientos y procesos afines (espuma y tapicería, en este caso); cuatro se ocupaban del ensamble de asientos (ubicadas en la zona centro-este del país, dos en el Estado de México, una en Puebla y otra en el Distrito Federal); dos plantas más realizaban la tapicería (CISA Saltillo y cISA Ramos Arizpe), y una (CISA Hermosillo) realizaba ensamblaje, soldadura, doblado y procesos de hule espuma (ELM Internacional, 1995).

Sin embargo, en 2000, Lear Corporation aumentó a 34 el número de plantas en todo el país (22 maquiladoras y 12 fabricantes directos). Es decir, en sólo cinco años la cantidad de plantas de esta empresa global pasó de diez a 34 (ELM Internacional, 2000; Lara, Trujano y García, 2005; García, 2004). En ese año, del total de maquiladoras, 19 se concentraban en una sola ciudad: Juárez, Chihuahua. Este salto cuantitativo impactó el nivel de ocupación: de poco más de 10 mil trabajadores y empleados registrados en 1995, después de cinco años eran 46 mil (ELM Internacional, 1995 y 2000).

El procedimiento específico por medio del cual Lear Corporation adquirió nuevas fábricas en México es el siguiente: primero compró acciones de una firma, después les

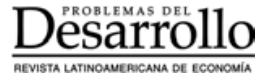


inyectó capital y finalmente las absorbió. Por este proceso han pasado Consorcio Industrial Mexicano de Autopartes (CIMA) y Central de Industrias S.A de C.V. (CISA). Por ejemplo, en 1995 había siete plantas con el nombre de CISA y Lear Corporation inicialmente sólo era socio; hoy son propiedad de dicho corporativo. Algo similar sucedió en el área de la maquila en 2000: Lear adquirió distintas fábricas del grupo FAVESA instaladas en México.

La expansión de este corporativo mediante el proceso de adquisiciones en nuestro país forma parte de una estrategia global de diversificación enfocada a conseguir nuevos recursos, mercados y tecnologías (Sako y Warburton, 1999). Ante la competencia entre empresas ubicadas en el mismo segmento, en este caso de asientos, y el aumento de la subcontratación de componentes por parte de las ensambladoras, los proveedores de autopartes tienen dos opciones: $a$ ) incrementar el valor agregado de los asientos, incorporando dispositivos de seguridad (bolsas de aire laterales, sistemas contra traumatismo cervical, diseños de piel, entre otros) y mejorar la comodidad de los mismos (reposabrazos, masajeadores lumbares, climatizadores de asiento), o $b$ ) expandir sus líneas de productos, lo cual implica avanzar hacia el diseño y fabricación de otros componentes o llegar, incluso, a cubrir la producción total de interiores. Para tal efecto pueden producir individualmente, realizar alianzas estratégicas o llevar a cabo fusiones y adquisiciones (Lara, Trujano y García, op. cit., y García, 2005). Lear Corporation no sólo está ampliando la producción del sistema de interiores mediante la integración vertical, sino que está ofreciendo a sus clientes nuevos diseños de asientos, los cuales cuentan con calefacción, dan masaje, son movibles, flexibles y resistentes. La forma en que se ha dado la integración vertical en Lear Corporation México se ejemplifica con el caso de la planta instalada en Tláhuac, Distrito Federal.

\section{La planta de Lear Tláhuac: origen y expansión}

Lear Corporation, para organizarse internamente, divide y especializa sus plantas tanto en la fabricación y ensamble del módulo de asientos y puertas, como del sistema de interiores. En 2004, la planta Tláhuac se dedicaba exclusivamente a producir respaldos, estructuras metálicas, fundas, cabeceras, poliuretanos, bastidores y subensambles de paneles para asientos y puertas. ${ }^{4}$ Sin embargo, Lear Tláhuac no siempre ha producido lo mismo; anteriormente era intensiva en fuerza de trabajo, debido a que existían las áreas de costura y corte, pero esas actividades fueron trasladadas a Lear Puebla para reorganizar sus propios espacios productivos y especializarse.

4 Esta planta inició actividades en octubre de 1969 con el nombre de cisA Tláhuac. En agosto de 1996, cambió su razón social a Lear Corporation (Lara, Trujano y García, 2005).

\section{DeSarrollo}


La necesidad de dicha transferencia surgió cuando el corporativo decidió comprar la empresa IPASA, uno de sus competidores locales, instalado en Cuautitlán Izcalli, el cual se dedicaba a producir viseras, sombrereras y toldos para Nissan. Después de la adquisición, parte de la maquinaria, equipo y personal que ahí laboraba fue trasladado a Lear Tláhuac. Mediante esa transferencia, esa planta comenzó a producir toldos automotrices, viseras, sombrereras y cubiertas para llantas y, de esa manera, se convirtió en proveedora de la ensambladora Nissan.

No obstante, para llevar a cabo el rediseño de la estructura organizacional y tecnológica de Lear Tláhuac y transferir los distintos procesos productivos desde IPASA, fue necesario que el corporativo coordinara múltiples habilidades y conocimientos y lograra la cooperación entre el personal de algunas de sus plantas instaladas en otras entidades del país. De acuerdo con un ingeniero entrevistado, que participó en dicho proceso de transferencia, a las instalaciones productivas de Tláhuac "llegó personal de las plantas de Estados Unidos, Ciudad Juárez, Monterrey, Hermosillo y Toluca. Asistieron ingenieros que apoyaron en el diseño y ubicación de la maquinaria, en mantenimiento, calidad y capacitación".

Mediante la concentración de los recursos especializados que Lear Corporation tenía dispersos en las distintas regiones del país se solucionaron, en menos de un mes, los problemas de adaptación y reconfiguración organizacional y productiva de la planta Tláhuac. La presión a la que se vio sujeta la movilización de recursos humanos, en un lapso tan corto, estaba asociada a que Lear Tláhuac tenía que asumir la responsabilidad de mantener las relaciones de proveeduría con Nissan, a fin de no perder su contrato.

Siempre que la situación lo amerita, los gerentes, ingenieros, supervisores y personal operativo son trasladados de una planta a otra con la finalidad de apoyar las distintas actividades del corporativo. Ese mismo proceso se presentó cuando Lear Tláhuac trasladó toda el área de costura a Lear Puebla. Para lograr ese objetivo, se movilizaron más de sesenta costureras, supervisores y trabajadores de capacitación desde el Distrito Federal.

Esta rotación del personal en el nivel interplanta no sólo se produce cuando es necesario solucionar un problema determinado, sino también para la capacitación laboral. Muchos ingenieros reciben cursos de calidad en Estados Unidos o en plantas instaladas en el país. Las redes de difusión de conocimiento y de las mejores prácticas entre las distintas plantas del corporativo se realizan de forma paralela a los vínculos comerciales, como se aborda en el siguiente apartado. 


\section{Las relaciones de Lear Tláhuac \\ con las otras plantas filiales y con el corporativo ${ }^{5}$}

Otra forma de colaboración intraempresa ocurre en el nivel comercial; en ese sentido, las plantas de Lear adoptan una relación de interdependencia de tipo mancomunada. ${ }^{6}$ Hasta 2002, Lear Tláhuac era el principal suministrador de costuras y tapizado para asientos de Lear Puebla. No obstante, las transacciones de esos productos cesaron cuando se observó que, al pasar la línea de costura de Tláhuac hacia la planta de Puebla, se reducían los costos en términos de tiempo y transporte, sobretodo porque el principal cliente de esta última unidad productiva es Volkswagen, aunque en la actualidad, solamente le envía fundas para asientos.

La planta de Tláhuac también ha sido cliente de la planta de Silao, Guanajuato. Le provee bastidores para que se tapicen; posteriormente, esta última se los vende a General Motors. A su vez, Lear Chihuahua se ha convertido en proveedor de insumos para Lear Tláhuac, al enviarle plásticos, alfombras y sistemas de arneses, mientras ésta produce uretanos para la planta de Puebla (véase Gráfica 1).

En relación con estos procesos de cooperación, un ingeniero industrial de la planta Toluca señaló: "Lear es una corporación, por lo tanto, nosotros podemos ser proveedores de otras Lear y otras Lear son proveedoras nuestras". Las relaciones de suministro de insumos observadas en las distintas plantas de Lear de México son coordinadas mediante precios de transferencia y en sustitución de precios de mercado. ${ }^{7}$

Como parte de su estrategia interna, también Lear Corporation México realiza un proceso de coordinación nacional de todas las plantas al concentrar la información financiera y autorizar o desaprobar las nuevas inversiones en maquinaria y equipo de las distintas unidades productivas (siempre que no se rebasen los topes presupuestales fijados por la dirección del corporativo).

5 La información de este apartado se obtuvo en 2004 durante la visita de campo, de las entrevistas realizadas a esta planta, así como de los archivos del acervo del corporativo.

6 Para Thompson (1994), la interdependencia mancomunada se produce cuando dos o más entidades comparten recursos y se ven unidas a un grupo, pero cada una de ellas mantiene su independencia y contribuye al conjunto. En ese caso, uno de los mecanismos de coordinación predominante es la estandarización de los productos y procesos, a fin de facilitar el intercambio y disminuir la incerti dumbre en la producción.

7 Al respecto, James Thompson resalta la importancia de los precios de transferencia como mecanismo interno de coordinación en las empresas, particularmente señala que: "los precios de transferencia se encuentran a menudo en formas de la variedad integrada de manera horizontal, en las que dos o más divisiones son secuencialmente interdependientes. La primera división vende su producto a la última, la cual compra su insumo desde dentro de la organización. Los precios de transferencia son un intento por sustituir de manera arbitraria el mecanismo faltante de mercado o el grupo de medida externo cuya ausencia se debe al diseño organizacional. Se espera que cada unidad involucrada se comporte como si fuera independiente con respecto a la otra" (op. cit::16).

\section{DeSarrollo}




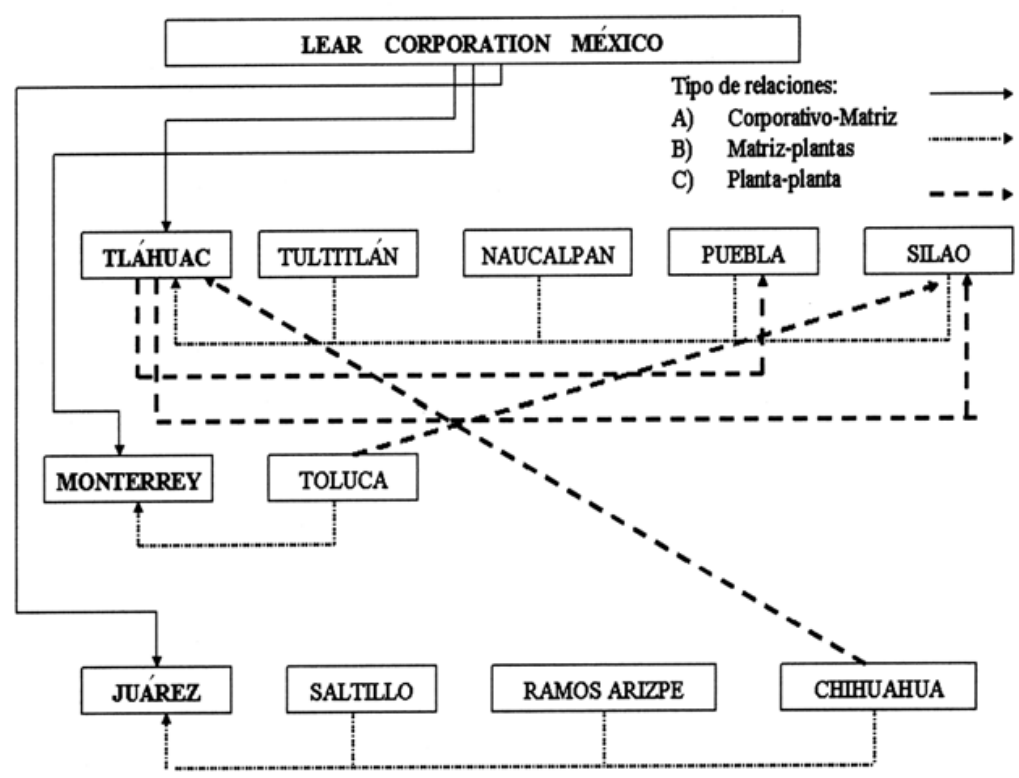

Gráfica 1. Estructura organizacional de las plantas que inegran Lear Corporation de México. Fuente: elaboración propia a partir de entrevistas.

Además, en el nivel regional, Lear ha establecido subcorporativos que supervisan y controlan la mejora en procesos y reducción de costos, coadyuvan a la solución de problemas y a la complementariedad productiva o tecnológica. A este respecto, se identificaron tres subcorporativos regionales: uno formado por las plantas de Toluca y Monterrey (coordinado por esta última), el cual básicamente fabrica los mismos productos, aunque el primero atiende al mercado interno y Monterrey al de Estados Unidos; otro subcorporativo regional está coordinado por la planta de Tláhuac e incluye a las de Tultitlán, Naucalpan, Puebla y Silao; el tercer grupo está coordinado por Ciudad Juárez e incluye Saltillo, Ramos Arizpe y Chihuahua (véase Gráfica 1). Cada planta goza de considerable autonomía para elegir proveedores, buscar clientes y nuevos proyectos; incluso, se da la competencia interplanta ${ }^{8}$ por desarrollar los proyectos que requieren las ensambladoras o ganar licitaciones. Sin embargo, a pesar de esa autonomía, el corporativo Lear mantiene intensa coordinación de todas sus actividades.

Lear Corporation es el único facultado para negociar los proyectos importantes, como la firma de acuerdos de cooperación para el desarrollo de un modelo de automóvil o la contratación de un proveedor global. También coordina todas las actividades de sus plantas, busca complementaridades entre ellas y apoyos en caso de necesidad, fija estándares tecnológicos y productivos, y las evalúa mensualmente (las califica en términos de

\footnotetext{
8 Respecto del proceso de cooperación y competencia en una industria véase García y Lara (2004).
}

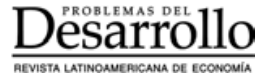


niveles de rotación del personal, accidentes de trabajo, inventarios, ausentismo de los trabajadores, entre otros).

Lear Corporation también coordina las mejoras tecnológicas realizadas en las distintas plantas. Si bien la investigación y desarrollo tecnológico se lleva a cabo en Estados Unidos, en caso de que alguna de las plantas ubicadas en nuestro país encuentre la forma de mejorar un producto o proceso, primero se tiene que presentar como proyecto, el cual puede o no ser aprobado por el corporativo de acuerdo con su factibilidad.

No obstante, Lear Corporation cuenta con una infraestructura informática denominada Lear Quality Tracking System (LQTS), la cual está instalada en todas las plantas. LQTS es una base de datos que recopila y comunica las mejoras registradas tanto en México, como en el nivel mundial. Se considera un sistema internacional de "lecciones aprendidas", en tiempo real, que ofrece a los empleados la posibilidad de monitorear y participar en la solución de problemas tecnológicos, productivos e ingenieriles. Para alimentarse, este sistema electrónico exige documentar los éxitos y fracasos obtenidos en proyectos específicos, así como difundir problemas y posibles soluciones. De acuerdo con esto, una vez que se haya resuelto un obstáculo administrativo, tecnológico o productivo, se requiere formalizar la metodología o los pasos que se siguieron, a fin de aplicarlos a los obstáculos similares que se presenten en otras plantas.

El esquema de coordinación de Lear México es complejo, ya que entre sus plantas existe cooperación comercial y redes de conocimiento, autonomía relativa para tomar decisiones no financieras, dependencia financiera y tecnológica respecto de las decisiones y apoyo del corporativo, además de competencia entre las filiales por ganar los proyectos de las ensambladoras y por mantener su propia rentabilidad.

Creemos que la posibilidad de que en una empresa como Lear se observen procesos tan complejos de coordinación — en el nivel interplanta — se explica por la capacidad de este corporativo para diseñar y dirigir a cada planta como un módulo organizacional, cuya característica principal es ser flojamente acoplado al sistema. ${ }^{9}$ En efecto, los procesos de modularidad se caracterizan porque se crea la capacidad para unir, mezclar e integrar módulos con características más o menos homogéneas y relativamente autónomos y, al mismo tiempo, mantiene ciertas interfases con el resto de los subsistemas (Baldwin y Clark, op. cit.).

Consideramos también que esta estructura de coordinación de tipo modular es uno de los elementos que permite a Lear Corporation México satisfacer las necesidades y adaptarse a las continuas exigencias de sus clientes.

Una característica de las estructuras organizacionales es su flojo acoplamiento: las partes o unidades que la integran se corresponden unas con otras, pero mantienen su identidad y especificidad, situación que implica considerar a la empresa o a un corporativo no como un ente monolítico, sino como heterogéneo o segmentado (Orton y Weick, 1990).

\section{DeSarrollo}


Cuadro 1

Principales productos de Lear Tláhuac por cliente durante 2003

\begin{tabular}{llll}
\hline Cliente & \multicolumn{1}{c}{ Producto } & \multicolumn{1}{c}{ Modelos } & Piezas por año \\
\hline General Motors & Bastidores para asientos & $805,806,820,830$ y 880 & 2.5 millones \\
& Cabeceras de asientos & 805 y 830 & 1.1 millones \\
& Bastidores de asientos & Monza, Joy y Swing & 540 mil \\
& Paneles para puertas & GMT 805/ 806 y 830 & 240 mil piezas \\
Daimler-Chrysler & Cabeceras para asientos & DR1500 & 350 mil \\
\cline { 2 - 3 } Ford & Bastidores de asientos & P-131 y h-215 & 15 mil \\
\hline
\end{tabular}

Fuente: información proporcionada por personal de Lear Tláhuac durante una visita a la planta.

Las relaciones de colaboración

entre Lear Tláhuac y las ensambladoras

Entre las principales ensambladoras a las cuales Lear Tláhuac surte componentes automotrices para distintos modelos se encuentran Ford — bastidores de asientos para sus plantas de Hermosillo y Cuautitlán-, General Motors — bastidores para asientos, cabeceras y paneles de puertas-, Daimler-Chrysler — cabeceras para asientos—y Nissan —lotes de viseras para automóviles una vez al mes-. Obsérvese que, en términos cuantitativos, la principal demanda es de su cliente General Motors (véase Cuadro 1).

Las relaciones de colaboración de Lear Tláhuac con Daimler-Chrysler (D-C), uno de sus principales clientes, iniciaron a principios de los noventa. En 1993 la planta, entonces denominada CISA, emprendió con esta ensambladora el Proyecto T-300 para apoyar el diseño y desarrollo de todos los asientos que en 1994 inició D-C para fabricar camiones ligeros (modelos BR-Body) en México y en Norteamérica. Las diferentes versiones que Lear Tláhuac elaboró para D-c fueron, entre otros, asientos y respaldos banca/vinil, asientos respaldos banca/tela, asientos y respaldos premium. Estos asientos se destinaron al mercado nacional (70\%) y a Estados Unidos y Canadá (30\% restante). Entre los requisitos que la ensambladora solicitó a Lear Tláhuac se encontraron: elaborar los prototipos, mostrar todos los reportes de las muestras pilotos que se aprobaron y lograr la certificación de calidad. De ahí que la planta Tláhuac haya alcanzado distintas normas de calidad productiva y ambiental, tales como QS-9000, ISO-14000 e ISO-TS16949; ${ }^{10}$ obtenidas en 1997, 2001 y a principios de 2004, respectivamente. ${ }^{11}$

10 Las normas de calidad internacional tienen como finalidad estandarizar los procedimientos y patrones de interacción comercial y productiva que en la industria automotriz se observa entre clientes y proveedores, tanto en el primero, como en los subsecuentes niveles jerárquicos que integran la cadena de valor. Los estándares reducen la variabilidad y contribuyen a reducir la incertidumbre y la complejidad tecnológica (Taboada, 2003).

11 Anteriormente, las ensambladoras asumían los costos por monitoreo en el área de calidad, al mandar parte de su personal a inspeccionar si las empresas autopartistas cumplían con los estándares

\section{DeSarrollo}


La planta Tláhuac aplica la filosofía de cero defectos. En caso de encontrarse alguno, éste se corrige analizando todas sus posibles causas, desde aquellas que se asocian con las materias primas hasta los errores humanos. A fin de tratar de ofrecer buena calidad en sus productos, también se utiliza six sigma, que es una técnica para reducir el nivel de variabilidad de los defectos localizados en las muestras de los productos supervisados al final de la línea de producción. ${ }^{12}$ Para abatir los defectos, en Lear Tláhuac, como en el resto de las plantas de Lear Corporation, se integran proyectos de solución de problemas que son liderados por los black belts (cinturones negros), quienes se encargan de coordinar al personal de las distintas áreas funcionales (ingeniería, calidad, materiales, recursos humanos y producción) para que, de manera multidisciplinaria, resuelvan los obstáculos. En caso de no detectar el problema, se pide apoyo al área de six sigma, que en el nivel nacional forma parte del corporativo, o a personal que integra el staff internacional (García, 2004). ${ }^{13}$

La designación de hombres garantía - trabajadores que pertenecen a Lear, pero que se encuentran en las instalaciones de sus clientes- es otra manera de asegurar que los asientos de Lear Tláhuac cuenten con las especificaciones de calidad estipuladas. Otras de sus tareas es corroborar que los productos terminados no sean dañados durante su traslado y solucionar los problemas que surjan en la instalación del asiento en la línea de ensamble, a fin de evitar el paro de la producción.

Es importante señalar el interés de Lear Tláhuac por responder a los requerimientos específicos de cada cliente. Por ejemplo, esta planta trabaja con el sistema justo a tiempo para la línea de asientos automotrices de la Ford Cuautitlán (en este caso, entrega los asientos en la línea de producción de la ensambladora). En el caso de la Nissan (ubicada en Japón), las relaciones comerciales son diferentes, ya que Lear Tláhuac mensualmente le manda viseras y otros productos.

De esta manera, una vez que Lear Tláhuac firma un contrato con algún cliente, tiene la obligación de responder a todas las contingencias que se presenten: "si, por ejemplo, Ford

fijados en el contrato. Hoy los costos de dicha supervisión han sido absorbidos por las empresas proveedoras de autopartes.

12 Seis sigma es "un concepto estadístico que mide un proceso en término de defectos. Alcanzar seis sigma implica que sus procesos presentan sólo 3.4 defectos por millón de oportunidades (DPMO), en otras palabras, están trabajando prácticamente de manera perfecta. Sigma (la letra griega) es un término en estadística que mide la llamada desviación típica. En su uso empresarial indica los defectos en las salidas de un proceso y nos ayuda a comprender cuánto se desvía el proceso de perfección (Brue, 2002:2).

13 Dentro del enfoque de gestión de six sigma participan diferentes categorías de empleados que se dedican a apoyar los proyectos de calidad. Entre los niveles en los cuales se dividen los participantes de seis sigma, de acuerdo con su nivel de jerarquía, se encuentran: champion (campeones), que son los directivos de nivel senior encargados de difundir esta metodología de calidad; master black belts (cinturones negros expertos), que son responsables de entrenar y guiar en la solución de problemas de calidad, y los black belts (cinturones negros), ingenieros que se dedican de tiempo completo a apoyar al master black belts (Brue, op. cit.).

\section{DeSarrollo}


nos dice que fabriquemos un bastidor, nosotros sabremos cómo encontrar la maquinaria y a los especialistas; es problema de nosotros", señaló un ingeniero de producción entrevistado en dicha planta. El prestigio y la reputación no sólo son con el cliente, sino también con el corporativo de Lear y el resto de las plantas con quienes mantiene una relación de cooperación, pero también de competencia. ${ }^{14}$

Una vez que el proceso de licitación de mercado le es favorable, los contratos que esta planta establece con sus clientes duran en promedio de cuatro a seis años y ello depende del ciclo de vida del modelo-año del auto para el cual se proveen los asientos automotrices.

Finalmente, es pertinente apuntar que como parte de la evaluación que las ensambladoras hacen a sus proveedoras, Lear Tláhuac es calificada continuamente mediante un sistema de puntaje que evalúa el sistema de entregas, calidad y reducción de costos de producción originalmente pactadas en el acuerdo firmado entre las partes. En su conjunto, las distintas formas de monitoreo que las ensambladoras hacen a las empresas de autopartes nos permite afirmar que las relaciones de coordinación y colaboración establecidas entre ambas partes no son de confianza ciega (García, Lara y Taboada, 2004).

\section{Conclusiones}

En la industria de asientos automotrices, las opciones de coordinación van desde la integración vertical hasta distintas formas de colaboración (fusiones, adquisiciones, alianzas tecnológicas estratégicas, contratos de transferencia de tecnología, acuerdos de subcontratación, entre otros), además de la alternativa de compraventa mediante precios, en el caso de los productos estandarizados.

Dada la complejidad tecnológica de los asientos (la heterogeneidad de los recursos y la diversidad de los conocimientos y habilidades) y el alto grado de competencia en el mercado, las coordinaciones inter e intraempresa se manifiestan como elementos centrales de la estrategia corporativa que siguen los líderes de esa industria. Un ejemplo de ese proceso es el de Lear Corporation, empresa global que inicialmente se dedicaba a la fabricación y ensamble de asientos y que hoy domina, además, el sistema de interiores; esta diversificación de las capacidades productivas y tecnológicas ha sido posible, entre otros factores, gracias a las relaciones de colaboración y a la integración vertical (por miedo de adquisiciones).

El caso de la planta de Lear-Tláhuac nos da una idea de la complejidad que implica la adquisición e incorporación de una nueva planta al sistema corporativo, en la cual la difusión de conocimientos y la aplicación de las habilidades acumuladas son fundamentales

14 Una vez que Lear Tláhuac termina un proyecto, compite con otros proveedores (tal es el caso de Johnson Controls) y también lo hace con el resto de las plantas del Corporativo de Lear México. La competencia es en relación con las capacidades tecnológicas, el nivel de especialización y la cercanía que se tenga con la ensambladora, de la cual es cliente potencial.

\section{DeSarrollo}


para tener éxito en la reestructuración y rediseño organizacional, pero también para diversificar la producción.

Entre los elementos de coordinación que resaltan en Lear México se encuentran los siguientes: el manejo de precios de garantía en el nivel interplanta, la movilidad de los recursos humanos, la cooperación entre filiales para la solución de problemas comunes, la existencia de un sistema de información por medio de intranet (LQTS) que documenta los procesos de aprendizaje y da seguimiento a resolución de problemas, la dirección y el apoyo financiero y tecnológico del corporativo, la capacitación continua del personal mediante la cual se difunden rutinas, la fijación de estándares y el establecimiento de subcorporativos regionales que coordinan, apoyan y supervisan a un conjunto de plantas.

Por otro lado, también se observa que en el corporativo de Lear México coexisten cooperación y competencia entre las distintas plantas. Esta dualidad contribuye al fortalecimiento de la relación entre ellas, pero al mismo tiempo promueve la obtención de mejores resultados competitivos en el corporativo.

En este trabajo se considera que las razones para explicar la cooperación y competencia interplanta y la relativa autonomía e independencia — que pueden mantener respecto del resto de las filiales e incluso del corporativo- es la estructura organizativa basada en módulos flojamente acoplados. En este caso, las plantas de Lear se consideran, por un lado, unidades de negocios que mantienen relativa autonomía y luchan por ser rentables y ganar proyectos en el mercado; pero, por otro lado, mantienen vínculos de colaboración dirigidos por el corporativo. La modularización organizacional adoptada por Lear permite que sus plantas se adapten a sus clientes y mantenga con ellos relaciones de colaboración en el largo plazo.

También es posible observar que en los procesos de coordinación interempresa, el cumplimiento en la certificación de distintas normas de calidad (ISO-9000, QS-9000, entre otros), el uso de la metodología y de la solución de problemas mediante six sigma, y el manejo de hombres garantía asignados por Lear Tláhuac para permanecer en las ensambladoras, revelan el interés central por satisfacer al cliente y por resolver los problemas de calidad que pudieran presentarse en la entrega de los módulos de asientos.

\section{Bibliografía}

Álvarez, Ma. de Lourdes, "Cambios en la industria automotriz frente a la globalización: el sector de autopartes en México", en Contaduría y Administración, núm. 206, julio-septiembre de 2002, pp. 29-49.
Aroq Limited, The Global Market for Automotive Interiors, Londres, Business Knowledge Providers, 2002.

Beecham, Matthew, Global Market for Automotive Seating, Londres, Business Knowledge Providers, 2001.

\section{DeSarrollo}


Baldwin, Carliss y Kim Clark, Design Rules, vol. I, Londres, The MIT Press, 2000.

Brue, Grez, Seis sigma para directivos, Madrid, MacGrawHill, 2002.

Cohen, Robert, "La reorganización internacional de la producción en la industria automotriz", en $E l$ Trimestre Económico, núm. 190, abril-junio de 1981, pp.383-404.

ELM International, The ELM Guide to Mexican Automotive Sourcing, MI: ELM International Inc., Washington, East Lansing, 1995.

- The ELM Guide to Mexican Automotive Sourcing, MI: ELM International Inc., 3a. ed., Washington, East Lansing, 2000.

Fine, Charles, El nuevo ciclo empresarial, Madrid, Paidós Empresa, 2000.

Freyssenet, Michel y Yannick Lung, "Between Globalization and Regionalization: What is the Future of the Automobile Industry?", en Actes $d u$ GERPISA, núm. 18, París, noviembre de 1996, http://www.univ-evry.fr/PagesHtml/laboratoires/ ancien-gerpisa/actes/index.html, en http:// imvp.mit.edu/pub99.html

García, Alejandro, "Coordinación inter e intra empresa en la industria automotriz de auto-partes: los casos de algunas plantas de Lear Corporation en México", tesis de doctorado en estudios organizacionales, México, UAM-Iztapalapa, octubre de 2004.

García, Alejandro y Arturo Lara, "Cluster y competencia (colaboración y competencia) industrial: algunos elementos teóricos por considerar", en Problemas del Desarrollo. Revista Latinoamericana de Economía, vol. 35, núm. 139, octubrediciembre de 2004, pp. 141-162.

García, Alejandro; Arturo Lara y Eunice Taboada, "La coordinación híbrida desde las perspectivas de Williamson y de Nooteboom", en Análisis Económico, vol. XIX, núm. 40, primer cuatrimestre de 2004, pp. 101-117.

Gereffi, Gary, "Globalización, cadenas productivas y pasaje de naciones a eslabonamientos superiores", en Jorge Basave et al. (coordinadores), Globalización y alternativas incluyentes para el siglo XXI, México, UNAM-FE-CRIM, 2002, pp. 89-134.

Helper, Susan et al., "Modularization and Outsourcing: Implications for the Future of Automotive Assembly", en Management of the Extended Enterprise Research Team, IMPV Publications Archive, 1999, http://imvp.mit.edu/ pubarcindx.html.
Lara, Arturo; Alejandro García y René Rivera, "La dinámica del cambio tecnológico en el segmento de asientos automotrices: el caso de Lear y Johnson Control Corporation", en Economía Teoría y Práctica, núm. 17, diciembre de 2002, pp. 3-30.

Lara, Arturo; Gerardo Trujano y Alejandro García, "Producción modular y coordinación en el sector de autopartes en México: el caso de la red de plantas de Lear Corporation", en Revista Región y Sociedad, núm. 32, vol. xVII, enero-abril de 2005, pp. 33-71, http://www.colson.edu.mx/ Region_y_Sociedad/revista2.asp? numero $=32$

Lear Corporation, Annual Report, 1999, en http:// www.lear.com.

—, Annual Report, 2000, en http://www.lear. com.

Okada, Yoshita, Competitive-cum-Cooperative: Inter-firm Relations and Dynamics in the Japanese Semiconductor Industry, Tokyo, Springer, 2000.

Orton, Douglas y Karl Weick, "Lossely Coupled System: a Reconceptualization", en Academy of Management Review, vol. 15, núm. 2, 1990, pp. 203-223.

Penrose, Edith, Teoría del crecimiento de la empresa, Madrid, Aguilar, 1962.

Sako, Mari y Warburton, Max, "MIT International Motor Vehicle Program: Modulariation and Outsourcing Project Interim of European Research Team", ponencia, International Motor Vehicle Program Annual Forum, Boston, 6-7 octubre de 1999, http://imvp.mit.edu/pubarcindx. html.

Shimokawa, Koichi, "Reorganization of the Global Automobile Industry and Structural Change of the Automobile Component Industry", en The International Motor Vehicle Program at MIT, 1999, working papers http://imvp.mit.edu/ pubarcindx.html.

Suzuki, Takao, "Age of the Automobile", en Journal of Japanese Trade and Industry, núm. 2, 1999, pp. 8-11.

Taboada, Eunice, "La credibilidad y la confianza frente al oportunismo en las relaciones de colaboración interfirma", en Gestión y Estrategia, núm. 12, julio-diciembre de 2003, pp. 197-205.

Thompson, James, Organizaciones en acción, México, McGraw Hill, 1994.

Womack, James; Daniel Jones y Daniel Roos, $L a$ máquina que cambio al mundo, Madrid, McGraw-Hill, 1990.

\section{DeSarrollo}

